\title{
Inductive ionospheric solver for magnetospheric MHD simulations
}

\author{
H. Vanhamäki \\ Arctic Research Unit, Finnish Meteorological Institute, Helsinki, Finland \\ visiting at: Solar-Terrestrial Environment Laboratory, Nagoya University, Nagoya, Japan \\ Received: 29 March 2010 - Revised: 12 August 2010 - Accepted: 29 December 2010 - Published: 10 January 2011
}

\begin{abstract}
We present a new scheme for solving the ionospheric boundary conditions required in magnetospheric MHD simulations. In contrast to the electrostatic ionospheric solvers currently in use, the new solver takes ionospheric induction into account by solving Faraday's law simultaneously with Ohm's law and current continuity. From the viewpoint of an MHD simulation, the new inductive solver is similar to the electrostatic solvers, as the same input data is used (field-aligned current [FAC] and ionospheric conductances) and similar output is produced (ionospheric electric field). The inductive solver is tested using realistic, databased models of an omega-band and westward traveling surge. Although the tests were performed with local models and MHD simulations require a global ionospheric solution, we may nevertheless conclude that the new solution scheme is feasible also in practice. In the test cases the difference between static and electrodynamic solutions is up to $\sim 10 \mathrm{~V} \mathrm{~km}^{-1}$ in certain locations, or up to $20-40 \%$ of the total electric field. This is in agreement with previous estimates. It should also be noted that if FAC is replaced by the ground magnetic field (or ionospheric equivalent current) in the input data set, exactly the same formalism can be used to construct an inductive version of the KRM method originally developed by Kamide et al. (1981).
\end{abstract}

Keywords. Ionosphere (Electric fields and currents) Magnetospheric physics (Magnetosphere-ionosphere interactions)

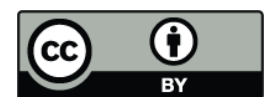

Correspondence to: $\mathrm{H}$. Vanhamäki (heikki.vanhamaki@fmi.fi)

\section{Introduction}

Magnetospheric magnetohydrodynamic (MHD) simulations are used extensively in studying the near-earth plasma environment, its inner dynamics and coupling with the solar wind. In addition to the magnetospheric (ideal) MHD model, simulations also require a separate ionospheric solver that provides inner boundary conditions for the magnetospheric solution.

It would appear that at the present time global MHD simulations use electrostatic solvers in the ionosphere (e.g. Janhunen, 1996; Raeder et al., 1998; Tanaka, 2000; Lyon et al., 2004, and references therein), meaning that inductive effects are ignored and the ionospheric electric field can be represented by a potential. Janhunen (1998) considered a type of electrodynamic solver where the ionospheric electric field may contain a non-potential component. However, even in this approach Faraday's law is not solved in the ionosphere, for the electric field is obtained by a direct mapping from the magnetosphere.

Coupling between magnetosphere and ionosphere can also be described in terms of Alfvén wave propagation and reflection at the ionospheric boundary. For example Lysak and Song $(2001,2006)$ and Waters and Sciffer (2008) have developed numerical models for solving linearized MHD wave equations in the inner magnetosphere, without resorting to separate ionospheric solvers. On the other hand the linearized wave models require pre-defined background magnetic field and density data. Recently Yoshikawa et al. (2010) have developed an Alfvénic magnetosphere-ionosphere coupling scheme, where the ionospheric boundary condition needed in MHD simulations is derived from reflection properties of shear Alfvén waves. However, such coupling schemes have not yet been implemented in present day simulations. 
The electrostatic approximation is usually valid in the ionosphere, because typical current systems evolve rather slowly, in time scales of several minutes. Nevertheless, it has been shown that inductive effects may play an important role in the reflection of Alfvén waves at the ionospheric boundary (e.g. Yoshikawa and Itonaga, 1996; Buchert, 1998; Lysak and Song, 2001). More recently Vanhamäki et al. (2007) showed that in very dynamical situations, like in a westward traveling surge, induction may contribute up to $30 \%$ of the total electric field in some limited areas. Takeda (2008) concluded that induction may affect rapidly changing, global current systems, such as the preliminary impulse of storm commencement. These results suggest that ionospheric induction may have a non-negligible role in magnetosphere-ionosphere coupling, especially during active periods such as substorm onsets.

In this article we present a scheme for an inductive ionospheric solver, where ionospheric Ohm's law, current continuity and Faraday's law are solved simultaneously and selfconsistently. From the viewpoint of an MHD simulation, the inductive solver is quite similar to the existing electrostatic solvers, as the same input data is used (field-aligned current [FAC] and ionospheric conductances) and similar output is produced (ionospheric electric field). However, the structure of the calculated electric field may be different, depending on the temporal evolution of the input data.

We begin by reviewing the electrostatic ionospheric solver presently used in MHD simulations in Sect. 2, together with some proposed alternative schemes. The theory behind the new inductive solver is discussed in Sect. 3, while in Sect. 4 we present some simple applications illustrating the feasibility of the method. Actual implementation of the new inductive solver in a magnetospheric MHD simulation is beyond the scope of the present theoretical study. Details of the solution algorithm are given in an Appendix.

\section{Background}

\subsection{Electrostatic solver}

The spatial grid resolution and time step in MHD simulations are limited by the Alfvén speed $\left(V_{\mathrm{A}}=|\boldsymbol{B}| / \sqrt{\mu_{0} \rho}\right)$ and Courant stability condition, which states that for a stable solution the time step must be smaller than the wave travel time across each grid cell. For this reason magnetospheric MHD simulations have an inner artificial boundary (AB), usually around 2-3 $R_{\mathrm{E}}$, as closer to Earth the increasing Alfvén speed would make full MHD solution computationally impractical (however, the linearized MHD wave equation can be solved all the way down to the ionosphere, see e.g. Lysak and Song, 2006; Waters and Sciffer, 2008). Instead, the magnetospheric simulation is coupled to an ionospheric solver by mapping FAC and electric field along magnetic field lines. Here we give a brief summary of the process, further details are given e.g. by Janhunen (1998).

The FAC distribution is calculated at the $\mathrm{AB}$ as

$j_{\|}=\hat{\boldsymbol{e}}_{\|} \cdot \nabla \times \boldsymbol{B} / \mu_{0}$,

where $\hat{\boldsymbol{e}}_{\|}$is the unit vector in the magnetic field direction. When mapped to the ionosphere $j_{\|}$scales with the flux tube cross section. Ionospheric conductivities are calculated using a pre-defined model of solar UV radiation and electron precipitation data estimated from the MHD variables at the AB (see e.g. Raeder et al., 1998; Janhunen, 1996).

The ionosphere is treated as a thin spherical shell, with height-integrated Hall, Pedersen and field-aligned conductances $\Sigma_{\mathrm{H}}, \Sigma_{\mathrm{P}}$ and $\Sigma_{0}$, respectively. The ionospheric Ohm's law is

$\boldsymbol{J}=\Sigma \cdot \boldsymbol{E}$.

Here $\boldsymbol{J}$ denotes the height-integrated horizontal current and we ignore the parallel component of the electric field. The ionospheric conductance tensor is (e.g. Brekke, 1997, chapter 7.12)

$\Sigma=\frac{1}{C}\left(\begin{array}{cc}\Sigma_{0} \Sigma_{\mathrm{P}} & -\Sigma_{0} \Sigma_{\mathrm{H}} \sin I \\ \Sigma_{0} \Sigma_{\mathrm{H}} \sin I & C \Sigma_{\mathrm{P}}+\Sigma_{\mathrm{H}}^{2} \cos ^{2} I\end{array}\right)$.

where $I$ is the inclination angle of the magnetic field $\left(+90^{\circ}\right.$ at the northern magnetic pole, $-90^{\circ}$ at the southern pole) and $C=\Sigma_{0} \sin ^{2} I+\Sigma_{\mathrm{P}} \cos ^{2} I$.

Current continuity means that

$\nabla_{\mathrm{h}} \cdot \boldsymbol{J}=j_{\|} \sin I$

where the subscript " $h$ " indicates that horizontal derivatives are calculated. Together with Ohm's law this gives us an elliptic differential equation for the ionospheric potential electric field $\boldsymbol{E}=-\nabla_{\mathrm{h}} \phi$,

$-\nabla_{\mathrm{h}} \cdot\left(\Sigma \cdot \nabla_{\mathrm{h}} \phi\right)=j_{\|} \sin I$.

The electric potential $\phi$ is mapped along magnetic field lines to the $A B$, where it is used as a boundary condition for the plasma velocity,

$\boldsymbol{V}=\boldsymbol{E} \times \boldsymbol{B} /|\boldsymbol{B}|^{2}$.

If an estimate of the potential drop between the magnetosphere and ionosphere is made, it can be added to $\phi$.

\subsection{Solution based on the electric field}

In principle it is possible to reverse the process described above: instead of FAC we calculate the electric field at the $\mathrm{AB}$ and map that to the ionosphere. Then it is a simple matter to solve FAC from ionospheric Ohm's law and map it back to the $A B$, where it is used to update the magnetic field of the MHD simulation. This kind of solver was considered by Janhunen (1998), who called it an electromagnetic solver, as 
the electric field mapped from the MHD simulation generally has a rotational part.

However, in this approach Faraday's law is not solved in the ionosphere and the rotational part of $\boldsymbol{E}$ is probably inconsistent with the time derivate of the magnetic field. So for the purposes of this study the solver suggested by Janhunen (1998) can be considered as electrostatic.

Janhunen (1998) identified two difficulties in this electric field-based solver: (1) mapping non-potential electric fields between magnetosphere and ionosphere is fundamentally ambiguous and (2) it is difficult to change the FAC at the $\mathrm{AB}$, as it affects the magnetic field inside the simulation, not only at the AB. Janhunen (1998) presented possible ways to overcome these difficulties, but nevertheless this approach has not been implemented in existing MHD simulations. The first problem is relevant also in the inductive ionospheric solver presented in Sect. 3 and is discussed there in more detail.

\subsection{M-I coupling with Alfvén waves}

In the above magnetosphere-ionosphere coupling schemes it is assumed that the electric potential and FAC are instantaneously mapped along (dipolar) magnetic field lines between the $\mathrm{AB}$ and ionosphere. In a more realistic description changes in the M-I system are transmitted as hydromagnetic Alfvén waves.

The Alfvén velocity varies considerably along the magnetic field line, from a few hundred $\mathrm{km} \mathrm{s}^{-1}$ in the ionosphere up to $10^{5} \mathrm{~km} \mathrm{~s}^{-1}$ at $1-2 R_{\mathrm{E}}$ altitude (e.g. Paschmann et al., 2002, Fig. 3.12). Consequently, the two-way travel time between $\mathrm{AB}$ and ionosphere could be order of $10 \mathrm{~s}$. This is longer than typical time steps of MHD simulations (especially if sub-cycling is used) and should therefore be included in the ionospheric solver. However, this propagation delay is ignored in the presently used electrostatic solvers discussed above, as well as in the new inductive solver presented in Sect. 3.

Reflection of Alfvén waves from non-uniformly conducting ionosphere with vertical background magnetic field was treated by Glassmeier (1984). He used the electrostatic approximation, where only shear waves are involved. If inductive effects are included, there is a mode conversion between shear and compressional Alfvén waves (e.g. Yoshikawa and Itonaga, 1996; Buchert, 1998; Lysak and Song, 2001). The mode conversion also depends on the inclination of the background magnetic field (Sciffer et al., 2004). More recently Lysak and Song (2006) and Waters and Sciffer (2008) have developed linearized MHD models of Alfvén wave propagation and reflection in the near Earth space.

The prospect of using an inner magnetosphere Alfvén wave model as an ionospheric solver in a global MHD simulation has been discussed by Yoshikawa et al. (2010). They developed a scheme for extracting the incident wave pattern from the MHD fields and updating the boundary con- dition at the $\mathrm{AB}$ using the reflected waves. Yoshikawa et al. (2010) considered only an electrostatic ionosphere (shear wave reflection) with vertical background magnetic field and ignored the propagation delay of Alfvén waves, but it might be possible to use a similar approach to connect e.g. the 3dimensional, fully electrodynamic Alfvén wave model developed by Lysak and Song (2006) to a global MHD simulation.

In the ionospheric reflection process both the electric field and FAC are modified, in contrast to the solvers discussed in Sects. 2.1 and 2.2, where either $j_{\|}$or $\boldsymbol{E}$ remains fixed. As the FAC is changed in the ionosphere, the problem of magnetic boundary condition at the $\mathrm{AB}$, discussed in Sect. 2.2, applies also to Alfvénic solvers.

\section{Inductive (electrodynamic) solver}

In this section we present a new inductive solver for magnetospheric MHD simulations. The new solver is very similar to the presently used electrostatic solvers discussed in Sect. 2.1, except that inductive effects are included in the ionospheric solution. While this means that the mapping between $\mathrm{AB}$ and ionosphere is still handled in a simplified manner, ignoring the wave propagation aspect of the coupling, it should also make the new solver more straightforward to implement in magnetospheric MHD simulations than the Alfvénic solvers discussed by Yoshikawa et al. (2010) and in the previous section.

The new solver uses the same input data as the electrostatic solver, $j_{\|}$calculated from the MHD solution and ionospheric conductance estimates. However in this case the electric field has a non-potential part, giving us one additional degree of freedom. We can write the electric field as

$\boldsymbol{E}=-\nabla \phi+\hat{\boldsymbol{e}}_{\mathrm{r}} \times \nabla \psi$,

where $\hat{\boldsymbol{e}}_{\mathrm{r}}$ is a unit vector in radial direction.

As we have one more unknown function than in the electrostatic case, we need one more equation. This is obtained by combining Faraday's and Ampere's laws,

$$
\begin{aligned}
& \nabla \times \boldsymbol{E}=-\frac{\partial \boldsymbol{B}}{\partial t}, \\
& \nabla \times \boldsymbol{B}=\mu_{0} \boldsymbol{j} .
\end{aligned}
$$

Here $\boldsymbol{j}$ is the 3 -D volume current, $\boldsymbol{j}=\delta\left(r-R_{\mathrm{I}}\right) \boldsymbol{J}+j_{\|} \hat{\boldsymbol{e}}_{\|}$, where $R_{\mathrm{I}}$ is the radius of the ionospheric shell.

Equations (2), (4), (8), (9) form a closed system that can be solved for the electric field, once the FAC and conductances are specified. This system is somewhat more complicated than the electrostatic case, as it involves either the second derivative of the electric field or integration of the magnetic field. One possible solution algorithm is developed in this study. 


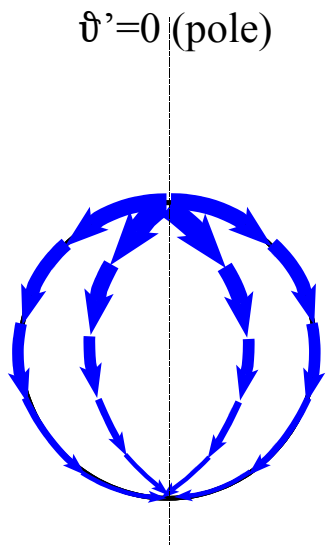

Curl-free elementary system

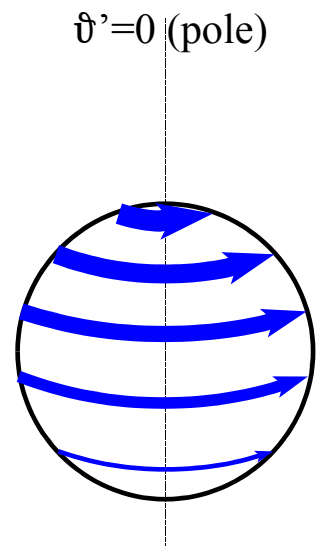

Divergence-free elementary system

Fig. 1. Curlf-free (left) and divergence-free (right) Spherical Elementary Current Systems (SECS). Figure provided by O. Amm.

\subsection{SECS-based solution algorithm}

Instead of the potentials $\phi$ and $\psi$ used in Eq. (7), we may equally well represent the electric field in terms of its divergence and curl (we only need the radial component of curl, as we use the thin shell approximation). The Spherical Elementary Current Systems (SECS) introduced by Amm (1997) offer a set of convenient basis functions for this kind of representation.

SECS are illustrated in Fig. 1. Each curl-free (CF) SECS represents a uniform source on a sphere plus an opposite delta-function source at the pole, while a divergence-free (DF) SECS has similar distribution of rotation. Mathematically speaking they are Green's functions of the $\nabla$. and $\nabla \times$ operators. Written in a spherical coordinate system $\left(r^{\prime}, \theta^{\prime}, \phi^{\prime}\right)$, with unit vectors $\left(\hat{\boldsymbol{e}}_{r^{\prime}}, \hat{\boldsymbol{e}}_{\theta^{\prime}}, \hat{\boldsymbol{e}}_{\phi^{\prime}}\right)$, having its pole at the center of the elementary systems, the vector fields are

$\boldsymbol{J}^{\mathrm{el}, \mathrm{cf}}\left(r^{\prime}, \theta^{\prime}\right)=\frac{I_{0}^{\mathrm{cf}}}{4 \pi R_{\mathrm{I}}} \cot \left(\frac{\theta^{\prime}}{2}\right) \hat{\boldsymbol{e}}_{\theta^{\prime}}$

$\boldsymbol{J}^{\mathrm{el}, \mathrm{df}}\left(r^{\prime}, \theta^{\prime}\right)=\frac{I_{0}^{\mathrm{df}}}{4 \pi R_{\mathrm{I}}} \cot \left(\frac{\theta^{\prime}}{2}\right) \hat{\boldsymbol{e}}_{\phi^{\prime}}$.

Here $I_{0}^{\mathrm{cf}}$ and $I_{0}^{\mathrm{cf}}$ are the scaling factors of the elementary systems. Together CF and DF SECS form a complete set of basis functions for representing any 2-D vector field on a sphere.

Let us define two grids in the ionospheric shell: $\boldsymbol{r}_{u}^{\mathrm{el}}=$ $\left(R_{\mathrm{I}}, \theta_{u}^{\mathrm{el}}, \phi_{u}^{\mathrm{el}}\right)$, where index $u=1 \ldots U$ give the points where the centers of the DF and CF SECS are placed, while $\boldsymbol{r}_{v}=$ $\left(R_{\mathrm{I}}, \theta_{v}, \phi_{v}\right), v=1 \ldots V$ are the points where we want to calculate the vector fields $\boldsymbol{E}$ and $\boldsymbol{J}$. For simplicity we assume that the div-free and curl-free SECS are placed at the same grid. In principle also the grid points $\boldsymbol{r}_{u}^{\mathrm{el}}$ and $\boldsymbol{r}_{v}$ may coincide, but often it is numerically beneficial to introduce two separate, interleaved grids.

With elementary systems we can calculate the horizontal current from its curl and divergence, as

$\mathfrak{J}=\mathbf{M}_{1} \cdot \mathfrak{d i v} \mathfrak{J}+\mathbf{M}_{2} \cdot \mathfrak{c u r l} \mathfrak{J}$

Here $\mathfrak{J}$ is a vector of length $2 V$ that contains the $\theta$ - and $\phi$ components of $\boldsymbol{J}$ at grid points $\boldsymbol{r}_{v}$,

$\mathfrak{J}=\left[J_{\theta}\left(\boldsymbol{r}_{1}\right), J_{\phi}\left(\boldsymbol{r}_{1}\right), J_{\theta}\left(\boldsymbol{r}_{2}\right) \ldots J_{\phi}\left(\boldsymbol{r}_{V}\right)\right]^{T}$.

The $U$-dimensional vectors $\mathfrak{d} \mathfrak{i v} \mathfrak{J}$ and $\mathfrak{c u r l} \mathfrak{l} \mathfrak{J}$ contain the divergence and curl of $\boldsymbol{J}$ at the $\boldsymbol{r}_{u}^{\mathrm{el}}$ grid points,

$$
\mathfrak{d i v} \mathfrak{J}=\left[\left.(\nabla \cdot J)\right|_{\boldsymbol{r}=r_{1}^{\mathrm{el}}},\left.\left.(\nabla \cdot \boldsymbol{J})\right|_{\boldsymbol{r}=\boldsymbol{r}_{2}^{\mathrm{el}}} \cdots(\nabla \cdot \boldsymbol{J})\right|_{\boldsymbol{r}=\boldsymbol{r}_{U}^{\mathrm{el}}}\right]^{T},
$$

$$
\mathfrak{c u r l} \mathfrak{l}=\left[\left.(\nabla \times J)_{\mathrm{r}}\right|_{\boldsymbol{r}=r_{1}^{\mathrm{el}}},\left.\left.(\nabla \times \boldsymbol{J})_{\mathrm{r}}\right|_{\boldsymbol{r}=\boldsymbol{r}_{2}^{\mathrm{el}}} \ldots(\nabla \times \boldsymbol{J})_{\mathrm{r}}\right|_{\boldsymbol{r}=\boldsymbol{r}_{U}^{\mathrm{el}}}\right]^{T}
$$

Here $\nabla \cdot \boldsymbol{J}$ and $(\nabla \times \boldsymbol{J})_{\mathrm{r}}$ should be interpreted as the average values over the grid cells. The components of vectors $\mathfrak{d i v} \mathfrak{J}$ and $\mathfrak{c u r l} \mathfrak{l} \mathfrak{J}$ (multiplied by the area of the grid cell) correspond directly to the scaling factors of the CF and DF SECS in Eqs. (10) and (11), respectively. Components of the transfer matrices $\mathbf{M}_{1,2}$ can be calculated using Eqs. (10) and (11), once the $\boldsymbol{r}_{u}^{\mathrm{el}}$ and $\boldsymbol{r}_{v}$ grids are specified. Details of forming the matrices are given in the Appendix.

In this article vectors like $\mathfrak{J}$ and $\mathfrak{d} \mathfrak{i} \mathfrak{v} \mathfrak{J}$ containing data from all grid points are written in fraktur font, in order to distinguish them from ordinary vectors, such as $\boldsymbol{r}$ and $\boldsymbol{J}$.

The divergent part of the current $(\mathfrak{d} \mathfrak{i v} \mathfrak{J})$ is known from the input FAC, while the rotational part $(\mathfrak{c u r l} \mathfrak{l} \mathfrak{J})$ is to be solved. We can write Ohm's law as

$\boldsymbol{E}=\mathbf{R} \cdot \boldsymbol{J}$,

where the resistance tensor $\mathbf{R}$ is obtained by inverting the conductance tensor in Eq. (3),

$\mathbf{R}=\frac{1}{\Sigma_{0}\left(\Sigma_{\mathrm{P}}^{2}+\Sigma_{\mathrm{H}}^{2}\right)}\left(\begin{array}{cc}C \Sigma_{\mathrm{P}}+\Sigma_{\mathrm{H}}^{2} \cos ^{2} I & \Sigma_{0} \Sigma_{\mathrm{H}} \sin I \\ -\Sigma_{0} \Sigma_{\mathrm{H}} \sin I & \Sigma_{0} \Sigma_{\mathrm{P}}\end{array}\right)$.

The curl and divergence of the inverted Ohm's law give us two relations between the electric field and current. In this case we need only the curl of $\boldsymbol{E}$, which can be written in terms of elementary systems as

$\mathfrak{c u r l e}=\mathbf{L}_{1} \cdot \mathfrak{d i v} \mathfrak{J}+\mathbf{L}_{2} \cdot \mathfrak{c u r l} \mathfrak{J}$.

Vector $\mathfrak{c u r l \mathfrak { E }}$ contains the curl of the electric field at the grid points $\boldsymbol{r}_{u}^{\mathrm{el}}$ and is completely analogous to the vector $\mathfrak{c u r l} \mathfrak{l} \mathfrak{J}$ defined in Eq. (15). Matrices $\mathbf{L}_{1,2}$ can be constructed using the previously defined matrices $\mathbf{M}_{1,2}$ and the inverted Ohm's law, see the Appendix for details.

We still need to write Faraday's law in terms of the SECS representation. It is simply

$\mathfrak{c u r l e}=-\frac{\partial \mathfrak{B r}}{\partial t}$, 
where the vector $\mathfrak{B r}$ contains the radial magnetic field at the grid points $\boldsymbol{r}_{u}$,

$\mathfrak{B r}=\left[B_{\mathrm{r}}\left(\boldsymbol{r}_{1}\right), B_{\mathrm{r}}\left(\boldsymbol{r}_{2}\right) \ldots B_{\mathrm{r}}\left(\boldsymbol{r}_{U}\right)\right]^{T}$.

The vector $\mathfrak{B \mathfrak { r }}$ can be written in terms of the current as

$\mathfrak{B r}=\mathbf{N}_{1} \cdot \mathfrak{d i v} \mathfrak{J}+\mathbf{N}_{2} \cdot \mathfrak{c u r l} \mathfrak{l} \mathfrak{J}$.

Matrices $\mathbf{N}_{1,2}$ can be obtained using the expressions for the magnetic fields of individual elementary systems, as outlined in the Appendix. In the case of vertical background magnetic field $\mathbf{N}_{1}=0$.

Now we can combine Eqs. (17), (18) and (20) as

$\mathbf{L}_{1} \cdot \mathfrak{d i v} \mathfrak{J}+\mathbf{L}_{2} \cdot \mathfrak{c u r l} \mathfrak{I}=-\frac{\partial\left(\mathbf{N}_{1} \cdot \mathfrak{d i v} \mathfrak{J}+\mathbf{N}_{2} \cdot \mathfrak{c u r l} \mathfrak{l}\right)}{\partial t}$.

As the divergent part of the ionospheric current, $\mathfrak{d} \mathfrak{i v} \mathfrak{J}$, is assumed to be known, the unknown rotational part $\mathfrak{c u r l} \mathfrak{l} \mathfrak{J}$ can be obtained by integrating this equation step-by-step in time. If we set the time-derivative in Eq. (21) to zero, we recover the electrostatic solver discussed in Sect. 2.1. After $\mathfrak{c u r} \mathfrak{r} \mathfrak{I}$ has been solved, the total current is obtained from Eq. (12) and the electric field from Eq. (16).

\subsection{Mapping rotational $E$ to the magnetosphere}

As mentioned in Sect. 2.2, the rotational induced part of the electric field does introduce some ambiguity to the mapping between ionosphere and magnetosphere, so the mapping procedure used in electrostatic solvers has to be modified.

Janhunen (1998) suggested a local potential mapping: In the vicinity of each ionospheric grid point $\boldsymbol{r}_{v}$ a local potential is defined as

$\Phi_{v}(\boldsymbol{r})=-\boldsymbol{E} \cdot\left(\boldsymbol{r}-\boldsymbol{r}_{v}\right)$.

This potential is then mapped to the $\mathrm{AB}$ along a few adjacent field lines, so that the electric field at the magnetic conjugate point of $\boldsymbol{r}_{v}$ can be evaluated. This procedure is repeated for each ionospheric grid point separately.

Another possibility is to simply ignore the rotational part of $\boldsymbol{E}$ : Define a global ionospheric potential by solving Poisson's equation

$\nabla^{2} \Phi=-\nabla \cdot \boldsymbol{E}$,

and map it to the $\mathrm{AB}$ as in the electrostatic case. It should be noted that usually the potential $\Phi$ defined here is not equal to $\phi$ obtained by solving Eq. (5). The physical justification for ignoring the rotational part of $\boldsymbol{E}$ may be obtained by considering the reflection of Alfvén waves at the ionosphere. The potential part of $\boldsymbol{E}$ is associated with shear waves, whereas the rotational part is connected to compressional waves (exactly true only for vertical $\boldsymbol{B}$, see e.g. Yoshikawa and Itonaga, 1996). Shear waves propagate only along the magnetic field, so the potential field is mapped directly to the AB. Most compressional waves generated in the reflection process are evanescent with exponentially decaying rotational $\boldsymbol{E}$. At high frequencies and for very large structures compressional waves can propagate in all directions, so in that case the rotational $\boldsymbol{E}$ experiences geometrical attenuation compared to the electrostatic $\boldsymbol{E}$. Therefore the rotational $\boldsymbol{E}$ at the AB can be neglected.

It may be necessary to determine the optimal mapping procedure trial-and-error tests, when coupling the proposed inductive solver to an magnetospheric MHD simulation, but that is beyond the scope of the present theoretical study.

\subsection{Inductive KRM algorithm}

In the above derivation we assumed that ionospheric conductances and FAC distribution (that is, vector $\mathfrak{d i v} \mathfrak{J}$ ) are given as the input data. However, it is worth noting that Eq. (21) can also be solved using the rotational part of the current $(\mathfrak{c u r l} \mathfrak{l} \mathfrak{J})$ as input. This gives us an inductive version of the KRM method originally developed by Kamide et al. (1981). At high magnetic latitudes the rotational current is equal to the ionospheric equivalent current obtainable from ground magnetic measurements (see e.g. Untiedt and Baumjohann, 1993).

Recently Vanhamäki and Amm (2007) developed a local version of the KRM method, where Cartesian elementary current systems (CECS, see Amm, 1997) formed the basis of the mathematical treatment. Apart from the different basis functions and somewhat different notation, the theory presented here is a generalization of the local KRM method. If we set the time derivative in Eq. (21) to zero and solve the system for the divergent current $\mathfrak{d} \mathfrak{i v} \mathfrak{J}$, we recover the solution presented by Vanhamäki and Amm (2007).

\section{Application examples}

Here we apply the solution algorithm developed in Sect. 3.1 to some realistic ionospheric current systems, including an omega-band model constructed by Amm (1996), using observational data obtained at northern Scandinavia by the Scandinavian Magnetometer Array, EISCAT radar and magnetometer cross, and STARE radar.

Our intent is to demonstrate that the theory developed in Sect. 3.1 can be used in practice. For this purpose it is sufficient to use mesoscale (order of $1000 \mathrm{~km}$ ) models of representative current systems, instead of coupling the ionospheric solver to a global MHD simulation. As Vanhamäki et al. (2007) has studied the ionospheric induction using the same omega-band models as employed here, we can use the previous results as a reference. However, it should be noted that Vanhamäki et al. (2007) used the potential part of the electric field as input data, whereas in the present study the FAC distribution is fixed. This difference will, in general, lead to different solutions, but the overall structure and magnitude of the induced electric field should be similar. 


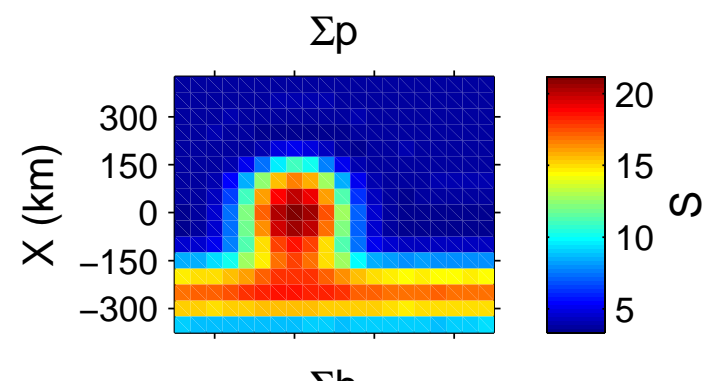

\section{$\mathrm{E}, \max =187 \mathrm{~V} / \mathrm{km}$}

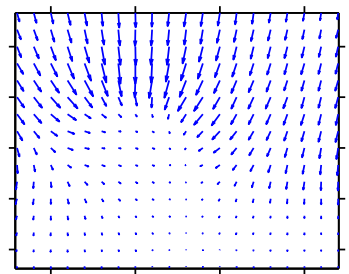

$\mathrm{J}, \max =2930 \mathrm{~A} / \mathrm{km}$
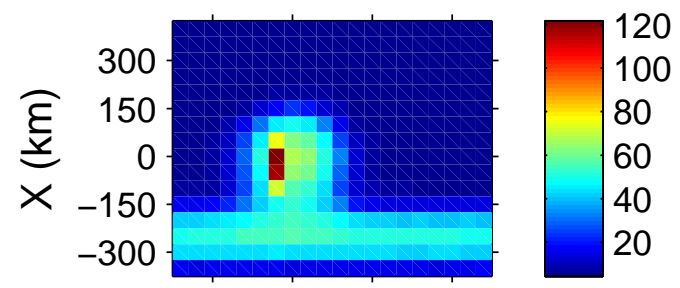

FAC

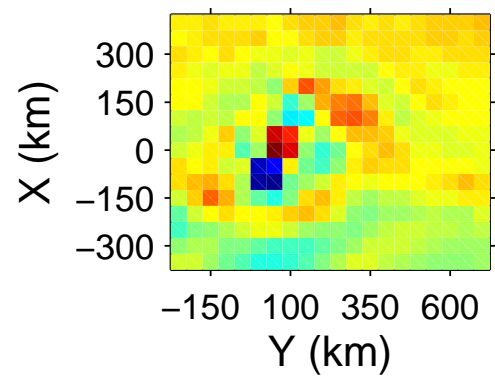

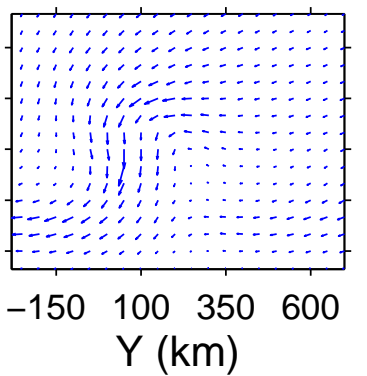

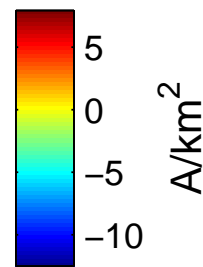
$\mathrm{Y}(\mathrm{km})$

Fig. 2. Model of an omega-band constructed by Amm (1996). Lefthand panels: Pedersen conductance $\Sigma_{\mathrm{P}}$, Hall conductance $\Sigma_{\mathrm{H}}$ and field-aligned current (FAC). Righthand panels: Electric field $\boldsymbol{E}$ and horizontal current $\boldsymbol{J}$.

It should be mentioned that in order to simplify these first calculations, we used Cartesian instead of spherical geometry, as the model areas are less than $1500 \mathrm{~km}$ across. We also neglected the tilt of the magnetic field lines. However, the theory presented in Sect. 3.1 and in the Appendix include also these effects, which are important in a global solution.

\subsection{Omega-band}

The omega-band model is illustrated in Fig. 2. Lefthand panels show the input variables $\Sigma_{\mathrm{P}}, \Sigma_{\mathrm{H}}$ and FAC used in the inductive solver. The electric and current fields obtained from the solver should be compared against the model variables shown in the righthand panels of Fig. 2.

The model shown in Fig. 2 is static, an instantaneous snapshot of the omega-band. We create temporal variations by moving the static model eastward at $2 \mathrm{~km} \mathrm{~s}^{-1}$, which is a high but still realistic speed (Paschmann et al., 2002, chapter 6).

Figure 3 shows results from the inductive solver. Lefthand panels shows the total electric field (sum of potential and rotational parts) together with associated horizontal and field-aligned currents. FAC is one of the input quantities in the inductive solver, so the result shown in Fig. 3 is identi- cal to the input model illustrated in Fig. 3, apart from small numerical inaccuracies.

The electric field and current obtained from the inductive solver are in good qualitative agreement with the original model, although their magnitude is too small by almost factor of 2. It is clear that the amount of electrojet type current flowing through the analysis area in East-West direction is severely underestimated, especially in the southern (bottom) side of the model. This behavior is even more evident in the 1-dimensional electrojet model discussed below. As for the electric field, largest errors occur in the low-conductance regions around the " $\Omega$ ", where errors in $\boldsymbol{J}$ are magnified in the inverted Ohm's law.

The righthand panels of Fig. 3 show the rotational (induced) part of the electric field solution, as well as the associated horizontal current and FAC. The induced electric field is strongest in the highly conducting "tongue" of the omega-band, where also the temporal changes are largest. The magnitudes of $\boldsymbol{E}^{\text {ind }}$ and $\boldsymbol{J}^{\text {ind }}$ are rather small, only about $0.8 \mathrm{~V} \mathrm{~km}^{-1}$ and $57 \mathrm{~A} \mathrm{~km}^{-1}$, respectively. However, as $\boldsymbol{E}^{\text {ind }}$ forms a locally closed current system in the high-conductance region, the associated $\mathrm{FAC}^{\text {ind }}$ is quite 


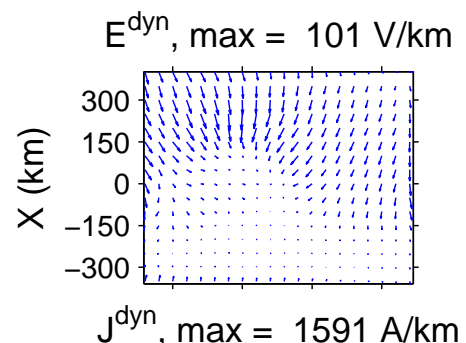

$E^{\text {ind }}, \max =0.8 \mathrm{~V} / \mathrm{km}$
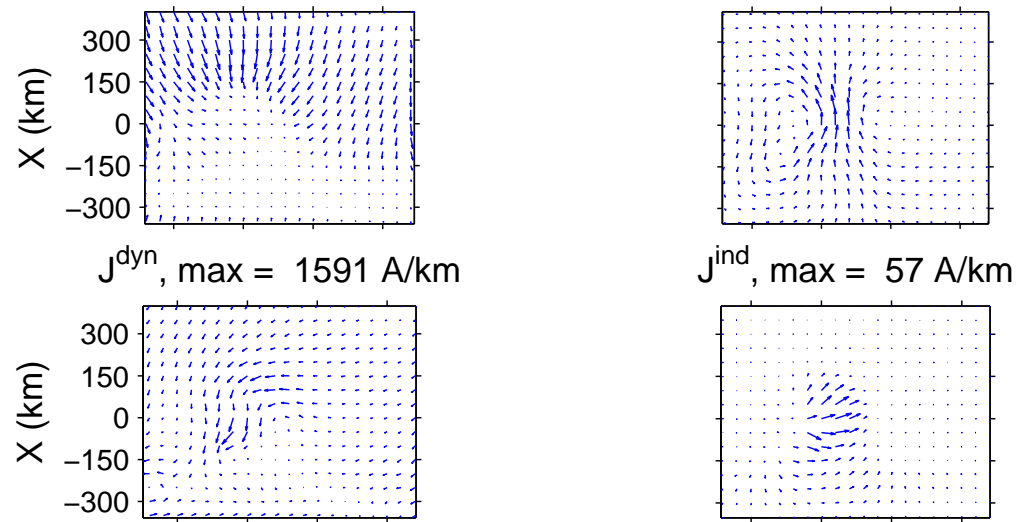

$F A C^{\text {dyn }}$

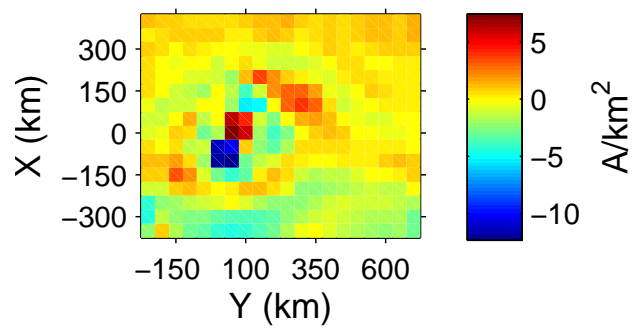

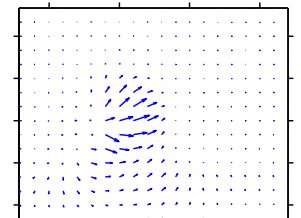

FAC ${ }^{\text {ind }}$

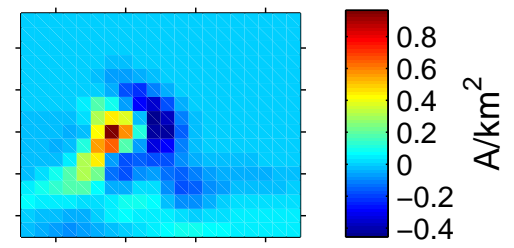

$-150100350600$

$\mathrm{Y}(\mathrm{km})$

Fig. 3. Results of the electrodynamic solver for the omega-band model. Lefthand panels: Total electric field $\boldsymbol{E}^{\text {dyn }}$, associated horizontal currents $\boldsymbol{J}^{\text {dyn }}$ and field aligned currents FAC ${ }^{\text {dyn }}$. Righthand panels: Induced part of the total electric field $\boldsymbol{E}^{\text {ind }}$ with associated currents $\boldsymbol{J}^{\text {ind }}$ and FAC ind

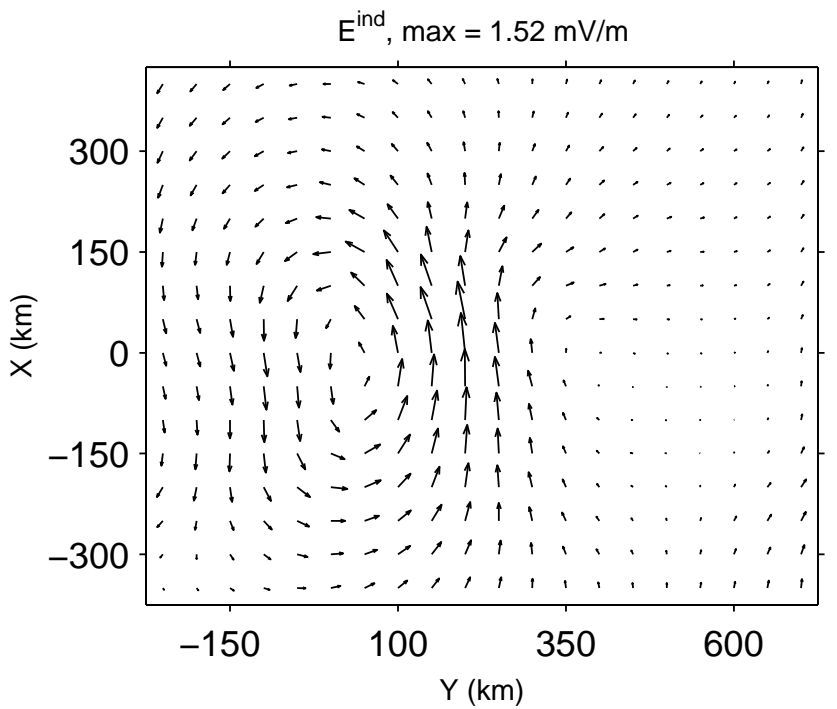

Fig. 4. Induced rotational electric field in the omega-band model calculated with the method of Vanhamäki et al. (2007).

significant, contributing up to $10 \%$ of the total FAC in the tongue.

The results shown in Fig. 3 should be compared against calculations by Vanhamäki et al. (2007), who used the potential part of the electric field as an input variable, instead of FAC used here. Figure 4 shows $\boldsymbol{E}^{\text {ind }}$ of the omega-band calculated by Vanhamäki et al. (2007). The result is very similar, almost identical to the one shown in the upper right panel of Fig. 3, apart from a factor $\sim 2$ difference in magnitudes. Similar magnitude difference is observed also in the WTS model (results not shown). The probable explanation is the different input data: In the present method the total FAC distribution is fixed, so the presence of $\boldsymbol{E}^{\text {ind }}$ changes also the potential electric field. Thus the induction effect is distributed by 2 degrees of freedom, the potential and rotational parts of $\boldsymbol{E}$. In the calculation presented by Vanhamäki et al. (2007) the potential electric field was fixed, so only the rotational $\boldsymbol{E}$ was affected by induction.

The total induction effect on the electric field is illustrated in Fig. 5. The upper panel shows the difference between the inductive (or electrodynamic) and static solutions, $\boldsymbol{E}^{\text {dyn }}$ and $\boldsymbol{E}^{\text {stat }}$, respectively, while the lower panel shows the relative effect. The static solution is obtained by neglecting the time derivative in Eq. (21). As mentioned above, the largest errors in the electric field solution are expected in low-conductance regions. Therefore, only those areas where $\left|\boldsymbol{E}^{\text {stat }}\right|>5 \mathrm{~V} \mathrm{~km}^{-1}$ and $\Sigma_{\mathrm{H}}>5 \mathrm{~S}$ are shown.

Figure 5 demonstrates that even though the rotational part of the electric field ( $\boldsymbol{E}^{\text {ind }}$ in Fig. 3) is quite small, induction may have much larger effect on the total electric field, up to $11 \mathrm{~V} \mathrm{~km}^{-1}$ or $20 \%$ in an omega-band. 


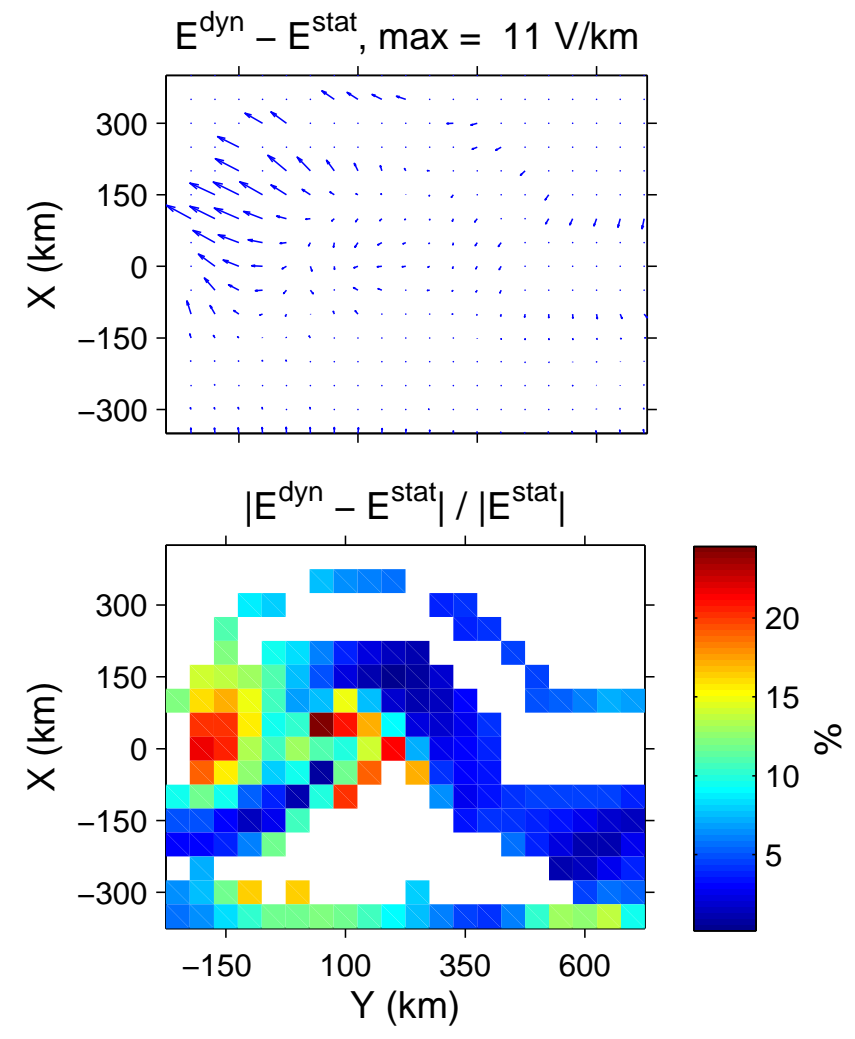

Fig. 5. Results for the omega-band model. Upper panel shows the difference between the electric fields obtained from static $\left(\boldsymbol{E}^{\text {stat }}\right)$ and electrodynamic $\left(\boldsymbol{E}^{\mathrm{dyn}}\right)$ solvers. Lower panel show the percentage difference in electric field magnitude. Only those areas where $\left|\boldsymbol{E}^{\text {stat }}\right|>5 \mathrm{~V} \mathrm{~km}^{-1}$ and $\Sigma_{\mathrm{H}}>5 \mathrm{~S}$ are shown.

We made similar calculation also with a WTS model (results not shown) employed by Vanhamäki et al. (2007). In this case time series was created by moving the model at $10 \mathrm{~km} \mathrm{~s}^{-1}$ westward, which is again in the upper range of realistic speeds. Here we present only a brief summary of the results.

The $\boldsymbol{E}^{\text {ind }}$ in a WTS produced by the inductive solver is very similar in structure to the results obtained earlier by Vanhamäki et al. (2007). The difference in magnitude is about factor of 2, same as in the omega-band case.

The electric field and horizontal current obtained as output from the inductive solver of Sect. 3.1 are in reasonable qualitative agreement with the input model and for $\boldsymbol{J}$ even the quantitative agreement is fairly good. The electric field is reproduced well in high-conductance areas, but in regions of low conductivity the solution is quite unreliable, probably due to the large contrast between high and low conductance regions (factor of 150 variations in $\Sigma_{\mathrm{H}}$, compared to 30 in the omega-band model) and boundary effects caused by the limited model area.

In absolute terms the induction effect is similar to the omega-band, close to $10 \mathrm{~V} \mathrm{~km}^{-1}$ in many places. The rel- ative change is $40-60 \%$ in many places, although in areas where $|\boldsymbol{E}|$ is small values exceeding $100 \%$ are observed.

\section{$4.2 \quad$ 1-dimensional electrojet}

In addition to the realistic, data-based models described above, we also tested the solver with a simple 1-dimensional electrojet. In this case the 1-D electrojet had a constant electric field and Gaussian conductance profile in the $\mathrm{x}$-direction and no variations in the $y$-direction. Temporal variations were not included.

This simple test case is worth mentioning because the SECS-based solution algorithm developed in Sect. 3.1 fails almost completely. The main reason for the failure is incorrect boundary condition. In the SECS-based approach we implicitly assume that divergence and rotation of $\boldsymbol{E}$ and $\boldsymbol{J}$ vanish outside the analysis area. In a 1-D electrojet it is possible to add a Cowling channel (Boström, 1964) to the system, without affecting the FAC used as input in the inductive solver. The only way to include the Cowling-type part to the solution is to impose explicit boundary conditions. However, this problem affects only regional analysis, as in global scales the solution is unique.

The SECS are intrinsically 2-dimensional, so 1-D structures and open current systems, where large part of the current flows through the analysis area, are difficult to model with them. This may explain part of the error in the omegaband results. Also this problem is expected to ease in global analysis, where all current systems are, by definition, closed.

\section{Discussion and conclusions}

The purpose of this article is to present a new inductive (or electrodynamic) ionospheric solver for magnetospheric MHD simulations. We use ionospheric conductances and FAC inferred from the the MHD simulation as the input data, similar to existing electrostatic solvers. In the new solver the internal induction in the ionosphere is taken into account by solving Faraday' law simultaneously with Ohm's law and current continuity.

The SECS-based solution algorithm presented in Sect. 3.1 is a modification of the inductive solver presented by Vanhamäki et al. (2006) and employed by Vanhamäki et al. (2007). The main difference is the type of input data used in the solver: Vanhamäki et al. (2006) assumed that the potential part of the ionospheric electric field is available, whereas here we use the FAC provided by a magnetospheric MHD simulation. Also the local elementary system -based KRM method developed by Vanhamäki and Amm (2007) is closely related to the solver presented in this article. In fact, as discussed in Sect. 3.3, the theory presented here includes also the KRM solution.

It is interesting to note that recently Takeda (2008) studied ionospheric induction using same input data and 
also somewhat similar numerical approach as developed here. Takeda (2008) solved a differential equation for the divergence-free current potential $\psi$ defined in Eq. (7), but presented the curl-free part of the current as a sum of simple vector systems equivalent to the CF SECS used here.

In Sect. 4 we present some preliminary results, that demonstrate the practical applicability of the new inductive solver. The results indicate that although the rotational part of the electric field is always local and rather small, about $1 \mathrm{~V} \mathrm{~km}^{-1}$ at most, it is usually concentrated in areas of high conductivity and therefore drives significant FACs. As the total FAC distribution is fixed, also the potential part of the electric field is indirectly modified by induction. In the omega-band and WTS models (results not shown) the difference in static and electrodynamic solutions may be $\sim 10 \mathrm{~V} \mathrm{~km}^{-1}$, or up to $20-40 \%$ of the total electric field.

Large inductive effects take place in the most dynamical ionospheric situations, such as during substorm onsets, which are important in magnetosphere-ionosphere coupling. We may expect that the difference between simulation runs using electrostatic and electrodynamic ionospheric solvers solutions increases with time, as the slightly different ionospheric solution affect the temporal evolution of the MHD simulation.

As discussed in Sect. 4, the output of the new solver is usually in good qualitative agreement with the correct $\boldsymbol{E}$ and $\boldsymbol{J}$ of the input model, but some errors are also present. Largest errors in $\boldsymbol{J}$ are probably related to the boundary conditions needed in regional analysis, so results of global analysis should be more reliable.

The electric field produced by the inductive solver includes a rotational part, which can't be mapped to the magnetosphere as simply as a potential field, as discussed in Sect. 3.2. One way to overcome the mapping problem would be to describe the magnetosphere-ionosphere coupling in terms of Alfvén wave propagation and reflection, like the model proposed by Lysak and Song (2006) discussed in Sect. 2.3. However, an Alfvénic model would be more complicated to couple to an MHD simulation than the inductive solver presented here, although recently Yoshikawa et al. (2010) have presented a possible coupling scheme.

The results presented in Sect. 4 indicate that the rotational part itself is rather small, but inductive change of the potential field is much larger. If this is true also in a global solution, and is not related to the problem of boundary conditions in regional analysis, then it may be feasible to simply ignore the rotational part of the electric field and map only the potential part to the MHD simulation.

In the present theoretical study we settled for presenting the theoretical basis of the new ionospheric solver and demonstrating its usability. Actual coupling of the new inductive solver to an magnetospheric MHD simulation is a topic for future studies.

\section{Appendix A}

In this Appendix we describe how to form the matrices $\mathbf{M}_{1,2}$, $\mathbf{L}_{1,2}$ and $\mathbf{N}_{1,2}$ defined in Eqs. (12), (17) and (20), respectively.

\section{A1 Matrices $\mathbf{M}_{1,2}$}

According to Eqs. (12)-(15) component $M_{1}^{(2 v-1, u)}$ of the matrix $\mathbf{M}_{1}$ gives that part of $J_{\theta}$ at location $\boldsymbol{r}_{v}$ that is contributed by a CF SECS located at $\boldsymbol{r}_{u}^{\mathrm{el}}$. Similarly, component $M_{1}^{(2 v, u)}$ gives curl-free part of $J_{\phi}$ at $\boldsymbol{r}_{v}$. Geometry of the situation is illustrated in Fig. A1. The vector field of each individual CF SECS is given in Eq. (10), where the scaling factor $V^{\text {cf }}$ corresponds to the components of vector $\mathfrak{d i v} \mathfrak{J}$. The remaining task is to convert the angle $\theta^{\prime}$ and unit vector $\hat{\boldsymbol{e}}_{\phi^{\prime}}$ defined in a SECS-centered coordinate system to the geographical system.

It is a straightforward exercise in spherical geometry to show that

$$
\begin{aligned}
& M_{1}^{(2 v-1, u)}=\frac{A_{u}}{4 \pi R_{I}} \frac{\cos \theta_{u}^{\mathrm{el}}-\cos \theta_{v} \cos \theta^{\prime}}{\sin \theta_{v}\left(1-\cos \theta^{\prime}\right)}, \\
& M_{1}^{(2 v, u)}=-\frac{A_{u}}{4 \pi R_{\mathrm{I}}} \frac{\sin \theta_{u}^{\mathrm{el}} \sin \left(\phi_{u}^{\mathrm{el}}-\phi_{v}\right)}{1-\cos \theta^{\prime}},
\end{aligned}
$$

where $A_{u}$ is the area of the grid cell centered at $\boldsymbol{r}_{u}^{\mathrm{el}}$ and

$$
\cos \theta^{\prime}=\cos \theta_{v} \cos \theta_{u}^{\mathrm{el}}+\sin \theta_{v} \sin \theta_{u}^{\mathrm{el}} \cos \left(\phi_{u}^{\mathrm{el}}-\phi_{v}\right) .
$$

Components of the matrix $\mathbf{M}_{2}$ are obtained in a similar fashion from the DF SECS defined in Eq. (10). The result is simply

$$
\begin{aligned}
& M_{2}^{(2 v-1, u)}=-M_{1}^{(2 v, u)}, \\
& M_{2}^{(2 v, u)}=M_{1}^{(2 v-1, u)} .
\end{aligned}
$$

\section{A2 Matrices $\mathrm{L}_{1,2}$}

Matrices $\mathbf{L}_{1,2}$ are defined in Eq. (17). Component $L_{1}^{(v, u)}$ of the matrix $\mathbf{L}_{1}$ gives curl of the electric field at point $\boldsymbol{r}_{v}$ caused by the CF SECS at $\boldsymbol{r}_{u}^{\text {el }}$, while matrix $\mathbf{L}_{2}$ is associated with the divergence-free elementary systems. It is straightforward to evaluate the curl of Eq. (16),

$$
\begin{aligned}
(\nabla \times \boldsymbol{E})_{\mathrm{r}}= & -\nabla R_{\theta \phi} \cdot \boldsymbol{J}-R_{\theta \phi} \nabla \cdot \boldsymbol{J}+\left(\nabla R_{\phi \phi} \times \boldsymbol{J}\right)_{\mathrm{r}}+ \\
& +R_{\phi \phi}(\nabla \times \boldsymbol{J})_{\mathrm{r}}-\frac{1}{R_{\mathrm{I}} \sin \theta} \frac{\partial\left(R_{-} J_{\theta}\right)}{\partial \phi},
\end{aligned}
$$

where components of the resistance tensor defined in Eq. (16) are abbreviated as

$$
\mathbf{R}=\left(\begin{array}{cc}
R_{\theta \theta} & R_{\theta \phi} \\
-R_{\theta \phi} & R_{\phi \phi}
\end{array}\right)
$$




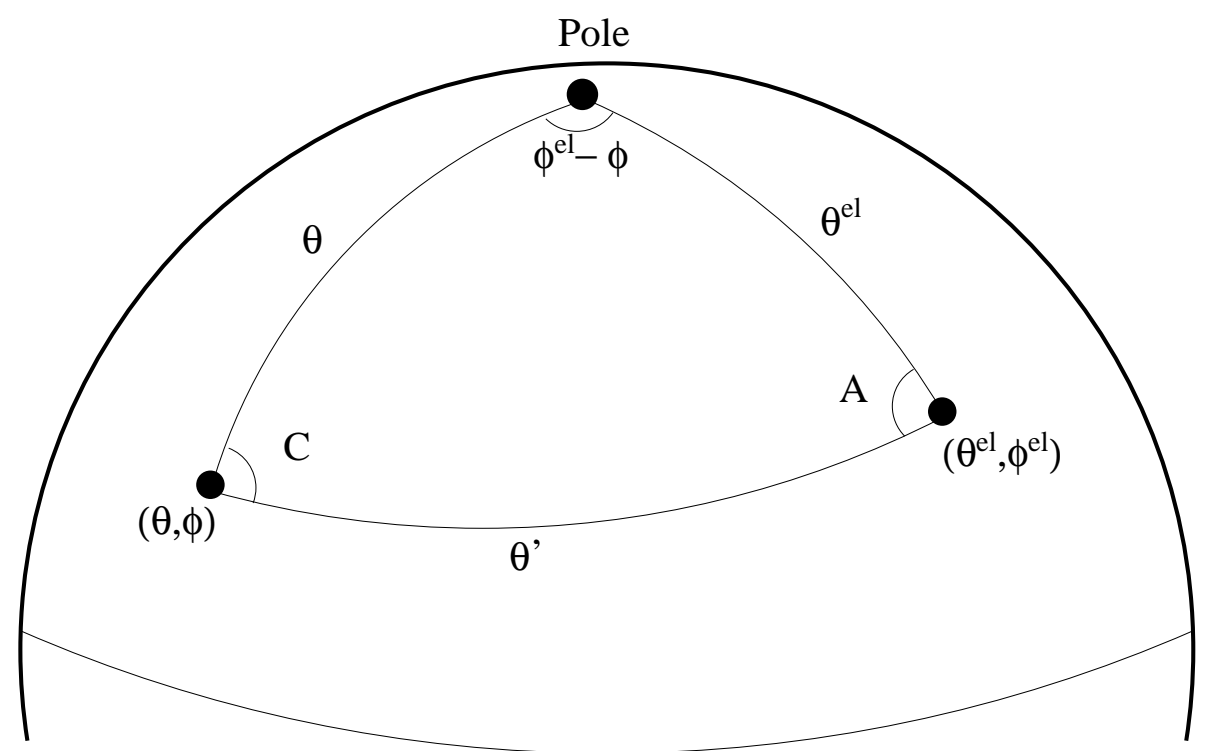

Fig. A1. Geometry of calculating components of matrices $\mathbf{M}_{1,2}$. The elementary system is located at $\left(\theta^{\mathrm{el}}, \phi^{\mathrm{el}}\right)$ and the vector field is evaluated at $(\theta, \phi) . \theta^{\prime}$ is the latitude of the point $(\theta, \phi)$ in the coordinate system centered at the elementary system.
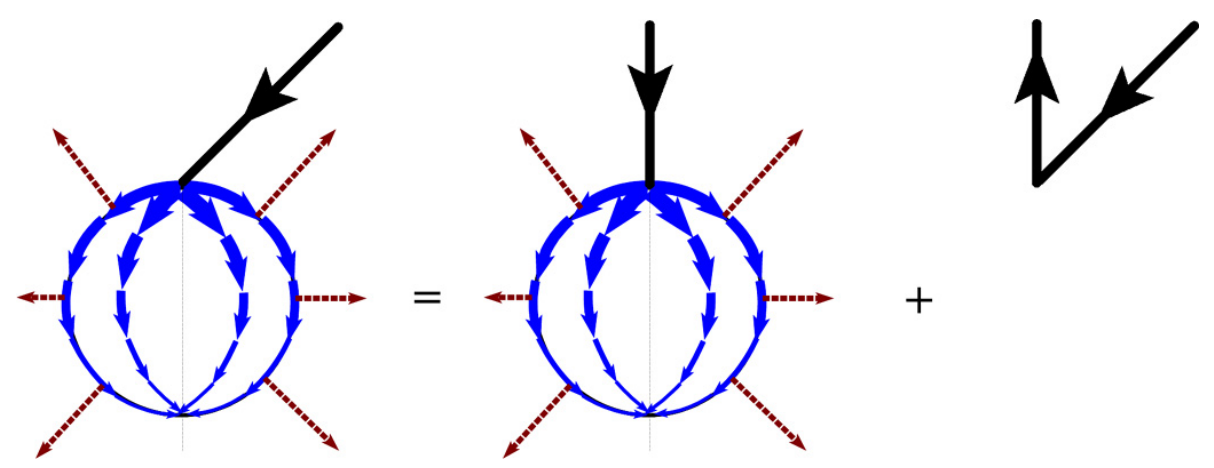

Fig. A2. Curl-free elementary system with tilted FAC at the pole. After Fukushima (1976).

and the difference of the diagonal components is

$R_{-}=R_{\theta \theta}-R_{\phi \phi}=\left(\frac{1}{\Sigma_{0}}-\frac{\Sigma_{\mathrm{P}}}{\Sigma_{\mathrm{P}}^{2}+\Sigma_{\mathrm{H}}^{2}}\right) \cos I$.

The divergence and curl of the DF and CF elementary systems defined in Eqs. (10), (11) are

$\nabla \cdot \boldsymbol{J}^{\mathrm{el}, \mathrm{cf}}=\left(\nabla \times \boldsymbol{J}^{\mathrm{el}, \mathrm{df}}\right)_{\mathrm{r}}=I_{0}\left(\delta^{2}\left(\boldsymbol{r}^{\mathrm{el}}-\boldsymbol{r}\right)-\frac{1}{4 \pi R_{\mathrm{I}}^{2}}\right)$,

$\left(\nabla \times J^{\mathrm{el}, \mathrm{cf}}\right)_{\mathrm{r}}=\nabla \cdot J^{\mathrm{el}, \mathrm{df}}=0$.

Components of $\mathbf{L}_{1,2}$ matrices can be deduced from Eqs. (12), (17), (A6) and (A9)-(A10) as

$$
\begin{aligned}
L_{1}^{(v, u)}= & -M_{1}^{(2 v-1, u)}\left(R_{\theta \phi ; \theta}+R_{\phi \phi ; \phi}\right)-M_{1}^{(2 v, u)}\left(R_{\theta \phi ; \phi}-R_{\phi \phi ; \theta}\right) \\
& -\frac{\partial\left(R_{-} M_{1}^{(2 v-1, u)}\right)}{R_{I} \sin \theta_{v} \partial \phi}-R_{\theta \phi}\left(\delta_{u, v}-\frac{A_{u}}{4 \pi R_{I}^{2}}\right), \quad \text { (A11) }
\end{aligned}
$$

$$
\begin{aligned}
L_{2}^{(v, u)}= & -M_{2}^{(2 v-1, u)}\left(R_{\theta \phi ; \theta}+R_{\phi \phi ; \phi}\right)-M_{2}^{(2 v, u)}\left(R_{\theta \phi ; \phi}-R_{\phi \phi ; \theta}\right) \\
& -\frac{\partial\left(R_{-} M_{2}^{(2 v-1, u)}\right)}{R_{I} \sin \theta_{v} \partial \phi}+R_{\phi \phi}\left(\delta_{u, v}-\frac{A_{u}}{4 \pi R_{\mathrm{I}}^{2}}\right) . \quad \text { (A12) }
\end{aligned}
$$

In the above formulas gradients are denoted as

$R_{\theta \phi ; \theta}:=\frac{1}{R_{\mathrm{I}}} \frac{\partial R_{\theta \phi}}{\partial \theta}$,

and so on. Function $\delta_{u, v}$ is defined so that

$\delta_{u, v}=\left\{\begin{array}{ll}1 & \text { if } \boldsymbol{r}_{v} \in \text { cell } \mathrm{u} \\ 0 & \text { otherwise }\end{array}\right.$,

and $A_{u}$ is the area of the grid cell centered at $\boldsymbol{r}_{u}^{\mathrm{el}}$.

\section{A3 Matrices $\mathbf{N}_{1,2}$}

Component $N_{1}^{(v, u)}$ of the matrix $\mathbf{N}_{1}$ gives the radial magnetic field at $\boldsymbol{r}_{v}$ caused by a CF SECS at $\boldsymbol{r}_{u}^{\mathrm{el}}$. Matrix $\mathbf{N}_{2}$ gives $B_{\mathrm{r}}$ 
associated with the DF SECS. When calculating the magnetic field of a CF SECS, the FAC associated with the divergence of the horizontal current has to be included. According to Eq. (A9), there is a delta-function FAC at the pole of the system and uniform, oppositely directed FAC elsewhere. We model the polar FAC as a tilted semi-infinite line current, while the uniform FAC is assumed to flow radially. The geometry of the uniform FAC need not be realistic, because we expect that in global scales the inward and outward flowing line currents will balance each other, and consequently the uniform FACs of the CF SECS will sum to zero.

Figure A2 illustrates the FAC distribution of a curl-free SECS and the way it can be decomposed into a purely radial FAC system plus a line current wedge. According to Fukushima's theorem the radial magnetic field of current system illustrated in the middle panel is zero (Fukushima, 1976). It is easy to show that the vertical line current of the current wedge also has $B_{\mathrm{r}}=0$. The remaining task is to calculate the radial magnetic field of the tilted semi-infinite line current.

Let us orient the primed, SECS centered coordinate system so that the line current flows along the zero meridian. The point $\left(\theta_{v}, \phi_{v}\right)$ where we want to calculate $B_{\mathrm{r}}$ is $\left(\theta^{\prime}, \phi^{\prime}\right)$ in the primed system (see Fig. A1). $\cos \theta^{\prime}$ is given in Eq. (A3) and

$\phi^{\prime}=A+D+180^{\circ}$,

where $D$ is the declination angle of the magnetic field (positive eastward). According to spherical trigonometry

$\sin A=\frac{\sin \theta_{v} \sin \left(\phi_{u}^{\mathrm{el}}-\phi_{v}\right)}{\sin \theta^{\prime}}$,

$\cos A=\frac{\cos \theta_{v}-\cos \theta^{\prime} \cos \theta_{u}^{\mathrm{el}}}{\sin \theta^{\prime} \sin \theta_{u}^{\mathrm{el}}}$.

The Biot-Savart law gives $B_{\mathrm{r}}$ as

$B_{\mathrm{r}}=\frac{\mu_{0} I^{\mathrm{el}, \mathrm{cf}}}{4 \pi} \int_{\text {line }} \frac{(\boldsymbol{d} \boldsymbol{l} \times \boldsymbol{V})_{\mathrm{r}}}{|\boldsymbol{V}|^{3}}$,

where $I^{\mathrm{el}, \mathrm{cf}}$ is the scaling factor of the CF SECS, $\boldsymbol{d} \boldsymbol{l}$ is directed along the line current and $\boldsymbol{V}$ is a vector from $\boldsymbol{r}^{\prime}=$ $\left(R_{\mathrm{I}}, \theta^{\prime}, \phi^{\prime}\right)$ to $\boldsymbol{d l}$. It is straightforward (although somewhat tedious) to express $\boldsymbol{d} \boldsymbol{l}$ and $\boldsymbol{V}$ in terms of the primed unit vectors at point $\boldsymbol{r}^{\prime}$ and evaluate the integral. In any case, the elements of the matrix $\mathbf{N}_{1}$ are

$N_{1}^{(v, u)}=-\frac{\mu_{0} A_{u}}{4 \pi R_{\mathrm{I}}} \frac{\sin \theta^{\prime} \sin \phi^{\prime} \cos I}{\alpha-\beta^{2}}\left(1+\frac{\beta}{\sqrt{\alpha}}\right)$,

where $I$ is the inclination of the magnetic field, $A_{u}$ is the area of the grid cell centered at $\boldsymbol{r}_{u}^{\mathrm{el}}$ and

$\alpha=2-2 \cos \theta^{\prime}$,

$\beta=\sin \theta^{\prime} \cos \phi^{\prime} \cos I+\left(1-\cos \theta^{\prime}\right) \sin I$.
Magnetic field of a DF SECS was calculated by Amm and Viljanen (1999). Components of the matrix $\mathbf{N}_{2}$ are obtained from the expression of the radial magnetic field,

$N_{2}^{(v, u)}=\frac{\mu_{0} A_{u}}{4 \pi R_{\mathrm{I}}}\left(\frac{1}{\sqrt{2-2 \cos \theta^{\prime}}}-1\right)$.

Acknowledgements. The work of $\mathrm{H}$. Vanhamäki is supported by the Academy of Finland (project number 126552). The author wishes to thank O. Amm, R. Fujii, A. Yoshikawa and A. Ieda for useful discussions and comments on the manuscript.

Topical Editor M. Pinnock thanks C. L. Waters and S. C. Buchert for their help in evaluating this paper.

\section{References}

Amm, O.: Improved electrodynamic modeling of an omega band and analysis of its current system, J. Geophys. Res., 101, 26772683, 1996.

Amm, O.: Ionospheric elementary current systems in spherical coordinates and their application, J. Geomagnetism and Geoelectricity, 49, 947-955, 1997.

Amm, O. and Viljanen, A.: Ionospheric disturbance magnetic field continuation from the ground to the ionosphere using spherical elementary current systems, Earth, Planets and Space, 51, 431440, 1999.

Boström, R.: A model of the auroral electrojets, J. Geophys. Res., 69, 4983-4999, 1964.

Brekke, A.: Physics of the upper polar atmosphere, John Wiley \& Sons, ISBN 0-471-96018-7, 1997.

Buchert, S.: Magneto-optical Kerr effect for a dissipative plasma, J. Plasma Phys., 59, 39-55, 1998.

Fukushima, N.: Generalized theorem for no ground magnetic effect of vertical currents connected with Pedersen currents in the uniform-conductivity ionosphere, Rep. Ionos. Space. Res. Japan, 30, 35-40, 1976.

Glassmeier, K.-H.: On the influence of ionospheres with nonuniform conductivity distribution on hydromagnetic waves, J. Geophys., 54, 125-137, 1984.

Janhunen, P.: GUMICS-3 - A global ionosphere-magnetosphere coupling simulation with high ionospheric resolution, ESA Symposium Proceedings on Environmental Modelling for Spacebased applications, ESTEC, Noordwijk, NL, 18-20 September 1996, ESA SP-392, 1996.

Janhunen, P.: On the possibility of using an electromagnetic ionosphere in global MHD simulations, Ann. Geophys., 16, 397-402, doi:10.1007/s00585-998-0397-y, 1998.

Kamide, Y., Richmond, A. D., and Matsushita, S.: Estimation of ionospheric electric fields, ionospheric currents, and fieldaligned currents from ground magnetic records, J. Geophys. Res., 86, 801-813, 1981.

Lyon, J. G., Fedder, J. A., and Mobarry, C. M.: The Lyon-FedderMobarry (LFM) global MHD magnetospheric simulation code, J. Atmos. Solar-Terr. Phys., 66, 1333-1350, 2004.

Lysak, R. and Song, Y.: A three-dimensional model of the propagation of Alfvén waves through the auroral ionosphere: First results, Adv. Space Res., 28, 813-822, 2001.

Lysak, R. and Song, Y.: Magnetosphere-ionosphere coupling by Alfven waves: Beyond current continuity, Adv. Space Res., 38, 1713-1719, 2006. 
Paschmann, G., Haaland, S., and Treumann, R. (Eds.): Auroral Plasma Physics, Space Sci. Rev., 103, 1-486, 2002.

Raeder, J., Berchem, J., and Ashour-Abdalla, M.: The geospace environment grand challenge: Results from a global geospace circulation model, J. Geophys. Res., 103, 14787-14797, 1998.

Sciffer, M. D., Waters, C. L., and Menk, F. W.: Propagation of ULF waves through the ionosphere: Inductive effect for oblique magnetic fields, Ann. Geophys., 22, 1155-1169, doi:10.5194/angeo22-1155-2004, 2004.

Takeda, M.: Effects of the induction electric field on ionospheric current systems driven by field-aligned currents of magnetospheric origin, J. Geophys. Res., 113, A01306, doi:10.1029/2007JA012662, 2008.

Tanaka, T.: The state transition model of the substorm onset, J. Geophys. Res., 105, 21081-21096, 2000.

Untiedt, J. and Baumjohann, W.: Studies of polar current systems using the IMS Scandinavian magnetometer array, Space Sci. Rev., 63, 245-390, 1993.

Vanhamäki, H. and Amm, O.: A new method to estimate ionospheric electric fields and currents using data from a local ground magnetometer network, Ann. Geophys., 25, 1141-1156, doi:10.5194/angeo-25-1141-2007, 2007.
Vanhamäki, H., Amm, O., and Viljanen, A.: New method for solving inductive electric fields in the non-uniformly conducting ionosphere, Ann. Geophys., 24, 2573-2582, doi:10.5194/angeo24-2573-2006, 2006.

Vanhamäki, H., Amm, O., and Viljanen, A.: Role of inductive electric fields and currents in dynamical ionospheric situations, Ann. Geophys., 25, 437-455, doi:10.5194/angeo-25-437-2007, 2007.

Waters, C. L. and Sciffer, M. D.: Field line resonant frequencies and ionospheric conductance: Results from a 2-D MHD model, J. Geophys. Res., 113, A05219, doi:10.1029/2007JA012822, 2008.

Yoshikawa, A. and Itonaga, M.: Reflection of shear Alfvén waves at the ionosphere and the divergent Hall current, Geophys. Res. Lett., 23, 101-104, 1996.

Yoshikawa, A., Nakata, H., Nakamizo, A., Uozumi, T., Itonaga, M., Fujita, S., Yumoto, K., and Tanaka, T.: Alfveniccoupling algorithm for global and dynamical magnetosphereionosphere coupled system, J. Geophys. Res., 115, A04211, doi:10.1029/2009JA014924, 2010. 